\title{
Germanica
}

\section{En quête du jeune homme idéal : L'affaire Maurizius de Jakob Wassermann et ses premiers critiques*}

Auf der Suche nach dem idealen Jungen: Jakob Wassermanns Der Fall

Maurizius und seine ersten Kritiker

\section{Pascale Avenel-Cohen}

\section{OpenEdition}

\section{Journals}

Édition électronique

URL : http://journals.openedition.org/germanica/2154

DOI : 10.4000/germanica.2154

ISSN : 2107-0784

\section{Éditeur}

Université de Lille

\section{Édition imprimée}

Date de publication : 30 juin 2002

Pagination : 41-59

ISBN : 9782913857070

ISSN : 0984-2632

\section{Référence électronique}

Pascale Avenel-Cohen, «En quête du jeune homme idéal : L'affaire Maurizius de Jakob Wassermann et ses premiers critiques* », Germanica [En ligne], 30 | 2002, mis en ligne le 16 juillet 2013, consulté le 06 octobre 2020. URL : http://journals.openedition.org/germanica/2154; DOI : https://doi.org/10.4000/ germanica. 2154

Ce document a été généré automatiquement le 6 octobre 2020.

(c) Tous droits réservés 


\title{
En quête du jeune homme idéal : L'affaire Maurizius de Jakob Wassermann et ses premiers critiques*
}

\author{
Auf der Suche nach dem idealen Jungen : Jakob Wassermanns Der Fall \\ Maurizius und seine ersten Kritiker
}

Pascale Avenel-Cohen

\section{NOTE DE L'AUTEUR}

* Ce texte a été présenté au colloque du GRAAL sur « Le culte de la jeunesse et de l'enfance en Allemagne 1870-1933 » à l'Université de Rennes II entre le 7 et le 9 mars 2002.

1 En févier 1928 parut la première édition du roman de Jakob Wassermann L'affaire Maurizius (Der Fall Maurizius) dont le succès immédiat ne se limita pas à l'Allemagne. Wassermann étant à l'époque un auteur bien établi, son dernier ouvrage fit l'objet de très nombreuses critiques dans la presse aussi bien littéraire que généraliste. Selon les priorités des journaux et revues et leur propre personnalité, les commentateurs mirent l'accent sur différents aspects du texte. Comme dans la grande majorité des publications d'après-guerre ${ }^{1}$, les deux axes majeurs qui se dégagent de la lecture de ces articles sont, d'une part, le problème de l'assimilation et de l'identité juive, plus ou moins développé selon le cas, d'autre part la justice, à laquelle tous les critiques consacrent une grande part de leurs propos, même ceux qui se contentent de résumer le roman et de l'encenser'2. Felix Weltsch constitue l'exception à la règle dans la mesure où sa critique aborde exclusivement le problème du judaïsme et de l'intégration, incarné dans le roman par le personnage de Waremme-Warschauer ${ }^{3}$. Cela ne signifie pas pour autant que la presse juive se soit détournée du problème de la justice, 
fondamental pour le judaïsme. Les titres des autres recensions le montrent, mais il ne m'a malheureusement pas été possible de localiser ces textes (cf. B. Bad-Strauss: "Justiz und Gerechtigkeit oder 'Der Fall Maurizius' », in Jüdische Rundschau, Berlin, 1928, Nr. 34 et Paul Wertheimer : "Jakob Wassermann und 'Der Fall Maurizius' », in Menorah, April 1928, Nr. 4, p. 205-206).

2 Lorsque les critiques ne concentrent leur analyse que sur un seul aspect du texte de Jakob Wassermann, il s'agit quasi systématiquement de la justice. Le cas de la Weltbühne , bien connue pour son combat dans ce domaine, est particulièrement flagrant : alors que le roman ne fait l'objet d'aucune recension, la Weltbühne en publie un court extrait dans lequel est dénoncée l'incompétence de certains juges («Ein Richter», in Die Weltbühne, 1928, Nr. 11 p. 399) à la suite d'un article de bientôt quatre pages consacré au procès Eisner et à son iniquité (Albert winter : "Der Rentenprozeß Eisner ", in Die Weltbühne, 1928, Nr. 11, p. 397-399). L'œuvre littéraire est ainsi instrumentalisée pour venir conforter les accusations du journaliste, tandis que l'attention du lecteur est attirée sur le texte de Wassermann. Erwin Pätzold ne pratique pas autrement dans le mensuel Eckart, mais après une longue série d'apostrophes au lecteur de L'Affaire Maurizius, dans laquelle il interroge son interlocuteur fictif sur la nature du droit: éthique ou juridique ${ }^{4}$. Tout comme Emil Hölcher dans Die Chistliche Welt ${ }^{5}$, il ne retient du texte de Jakob Wassermann, auquel il adhère totalement, que la volonté de nourrir le débat sur la justice dans le climat difficile de la République de Weimar où les scandales judiciaires se succèdent. Cette focalisation extrême sur ce seul aspect du roman s'explique par le fait que le critique voit dans le fossé croissant entre l'appareil judiciaire de la République de Weimar et la justice ("Gerechtigkeit und Justiz») la « tragédie du peuple allemand " (« unsere Tragödie, die Tragödie unseres Volkes »). Ce faisant, il transforme le jeune Etzel Andergast en un personnage monolithique : « Il est le principe de pureté et de vérité, qui vainc les paragraphes encroûtés, le principe du droit éternel et rayonnant, qui peut certes être obscurci, mais ne peut pas disparaitre " ( Er ist das Prinzip der Reinheit und Wahrheit, das über die knöchernen Paragraphen siegt, das Prinzip des ewigen leuchtenden Rechts, das wohl verdunkelt werden kann, aber nicht untergehen »).

3 Les autres articles dont la recension est axée essentiellement sur le problème de l'adéquation ou inadéquation entre le concept de justice et le système judiciaire de la République de Weimar sont généralement plus nuancés, à l'instar de Kurt Martens dans Die Literatur ou de Wolfgang von Einsiedel dans Die schöne Literatur ${ }^{6}$ qui n'hésite pas à dénoncer vivement ce qu'il considère comme de la facilité inconséquente, car, écrit-il, il est aisé de dénoncer les erreurs de la justice si on laisse dans l'ombre ses difficultés et ses mérites. La véhémence dont il fait preuve dépasse de loin les déclarations pondérées du juge Karl Doppfel, dans sa réponse à Emil Hölcher (Karl Doppfel: « Nochmals : Der Fall Maurizius. Zu dem Aufsatz in Nr. 16 », in Die Christliche Welt, 6. Okt. 1928, Jg. 42, Nr. 19, Gotha, p. 910-913). Il est vrai que ce dernier ne juge que les conclusions générales du critique chrétien sur la justice, tandis que Wolfgang von Einsiedel fait acte de critique littéraire. Ce faisant, il est le seul à dénier au roman toute qualité littéraire, politique ou sociale ${ }^{7}$.

4 S'agissant des ouvrages et articles parus après 1945, ils se caractérisent par la même bipolarité entre la critique de la justice et le problème de l'assimilation. Exception faite d'une toute petite minorité de textes ${ }^{8}$, la jeunesse du personnage, si elle est abordée, n'est considérée que comme secondaire. Pourtant dans sa recension du 2 mars 1928 
publiée dans la Literarische Welt ${ }^{9}$, Max Rychner fait du texte de Wassermann un hymne à la jeune génération.

Or, à la lecture du roman, une telle réaction ne peut manquer de surprendre. En effet, contrairement à ce que pourrait laisser supposer cette approche, le protagoniste n'est pas le seul personnage jeune du texte et les autres ne sont pas tous valorisés, loin s'en faut. $\mathrm{Si}$, en règle générale, Wassermann ne leur accorde que peu de cas, s'agissant des camarades de classe de Etzel par exemple, d'autres personnages, tels le jeune Paalzow (p. 240-241 $)^{10}$, rappellent au lecteur que le jeune héros est une exception.

6 Il est d'ailleurs frappant de constater que les qualités sur lesquelles Max Rychner se focalise, à savoir "l'intuition du cœur» («Intuition des Herzens»), l'illumination ( Erleuchtung »), le « cœur ardent » («brennendes Herz »), pour finir par « la flamme claire du cœur de jeune homme de Etzel» («die klare Flamme in Etzels Jünglingsherzen »), sont des qualités que le jeune personnage partage principalement avec sa mère et sa grand-mère. Dans les pages consacrées à la Générale, la mère du procureur Andergast, père du jeune Etzel, l'auteur insiste sur le fait qu'elle ne porte pas ses soixante-treize ans (p.40) et qu'elle rougit comme une jeune fille («wie ein Backfisch » p. 40) à tout compliment. Plus loin, Wassermann insiste sur sa ressemblance frappante avec son petit-fils en dépit d'une différence d'âge de cinquante sept-ans («Sie sah in diesem Augenblick dem um siebenundfünfzig Jahre jüngeren Enkel außerordentlich ähnlich», p. 45). La mère du jeune Etzel, Sofia, chassée par son mari avec interdiction de revoir son fils, alors âgé de six ans, a elle aussi une apparence étonnamment juvénile ("[ihre] Züge [hatten] eine überraschende Jugendlichkeit bewahrt », «Daß Sofia so unerwartet jung aussah... » p. 411). Elle dispose en outre de cette intuition du cœur ("Erleuchtung des Herzens » p. 412), de « l'instinct aiguisé » de son fils qui fait tant défaut à son ex-mari (« mit ihrem geschärften Instinkt » p. 419), et, dans ses yeux, brille la même étincelle («Funkeln» p.412) que dans ceux de Etzel (p. 11).

7 Or, dans son article, Max Rychner appuie toute sa description de l'adolescent sur la métaphore du feu. Il ne fait en cela que reprendre l'image de la lanterne sourde («Blendlaterne » p. 133), l'un des leitmotivs du roman, symbole de l'intuition de Etzel, de son attirance infaillible pour la justice, qui deviendra une image obsessionnelle chez son père, le procureur Andergast : «Le miroir lui montre une sorte de David debout dans le creux de la main d'un Goliath projettant la lumière de sa lanterne sourde à travers ce cerveau d'une effrayante similitude avec un escargot» («Der Spiegel zeigt ihm eine Art von David, der auf der flachen Hand eines Goliaths steht und mit der Blendlaterne das schaurig schneckengleiche Gehirn durchleuchtet » p. 533, ou p. 259). Mais tandis que dans ce feu Max Rychner voit une capacité supérieure propre à la flamme de la jeunesse, d'autres critiques, tels Victor Zuckerkandl dans la Neue Rundschau ${ }^{11}$ ne distinguent dans cette métaphore que l'instinct aveugle et naïf de l'enfance ("Kindliche Naivität des Knaben », in NR, 1928, p. 430). Il est à ce propos l'un des rares à présenter Etzel comme un "enfant " plutôt que comme un "jeune " ( "Junge ») avec Fritz Engel, le critique du Berliner Tageblatt (cf. note 2).

De détentrice d'un pouvoir inaccessible aux adultes qu'elle était pour Max Rychner dans les colonnes de la Literarische Welt, la jeunesse n'est plus amenée à distinguer l'injustice de la justice que par défaut dans la Neue Rundschau. À cet égard, Victor Zuckerkandl ne laisse pas planer la moindre ambiguité. Selon son analyse, l'instinct qui pousse Etzel vers la vérité n'est ni « une mystérieuse intuition », ni une découverte ou 
une science, qui lui permettrait de voir au-delà de l'horizon des adultes; ce sont au contraire son manque de maturité et son inexpérience de la vie qui confèrent à son regard une sorte de pré-science. Il voit là où les plus âgés sont aveugles car il n'est pas encore familiarisé avec «l'habitude » et «l'utilitarisme » liés aux responsabilités de la vie quotidienne. Dans cette perspective, l'adolescent n'est pas doté de vertus supérieures, il n'est qu'un homme encore inachevé qui tire avantage, dans un domaine précis, d'une propriété éphémère. La lucidité si frappante du jeune Etzel, cette lanterne sourde dont la lumière transperce jusqu'au cerveau de son père, n'est plus ici qu'un regard « infantile».

Es ist keine Erkenntnis auch keine geheimnisvolle Intuition, es ist ganz einfach der Blick des Kindes, für den die Gitter, in die Zweck und Gewohnheit das Lebendige sperren, nicht vorhanden sind. Da ist er, Etzel, das Kind, der Mensch ohne Trägheit des Herzens. (Die Neue Rundschau, 1928 p. 427)

Ce n'est pas une connaissance, pas même une intuition mystérieuse, c'est tout simplement le regard de l'enfant pour lequel les grilles, derrière lesquelles l'utilitarisme et l'habitude enferment la vie, n'existent pas. Tel est Etzel, l'enfant, l'homme sans paresse du cœur.

Dans une telle perspective, le cheminement de Etzel est ramené à un parcours somme toute assez simpliste : étant lui même un être élémentaire, il finit par rejoindre le sein de la nature incarné par sa mère : «Er ruft nach der Mutter : neue Bindung an die Natur soll ihm Kraft geben » (Die Neue Rundschau, 1928, p. 430).

10 À la lecture de cette interprétation, il serait aisé de conclure de ces correspondances entre l'instinct de Etzel et celui de sa mère que Wassermann n'a fait que succomber au préjugé commun au dix-neuvième siècle selon lequel les hommes auraient été fondamentalement guidés par l'intellect, tandis que les femmes et les enfants se seraient laissé aller aux élans de leur cœur. Il est certain que ce stéréotype joue un rôle dans le roman, même si Victor Zuckerkandl force largement le trait. Il suffit pour s'en convaincre d'étudier le vocabulaire employé pour caractériser Etzel.

11 Systématiquement cet adolescent de seize ans est appelé un enfant (« ein Kind»), un gamin («Der Bub» ou «Der Knabe») éventuellement haut comme trois pommes (« Dreikäsehoch» p. 481), ou, plus rarement, un jeune garçon (« Der Junge ») au mieux « ein Jüngling» (p. 105, p. 484) et une seule fois « Der Halbwüchsling» (p. 424) et « das Halbkind» (p. 475), deux termes guère valorisants décrivant un individu dont la croissance n'est pas achevée. Parvenu au terme de son initiation, quatre lignes avant la fin du roman, Etzel Andergast reste un enfant, comme l'indique sa voix («Bubenstimme » p. 539) ${ }^{12}$.

12 Il serait toutefois trompeur de ne voir dans le roman qu'une illustration de la nature instinctive de la jeunesse. Le traitement du personnage de Anna Jahn en est la preuve. Jeune femme de dix-neuf ans éclatante de beauté au moment du crime dont est accusé à tort Maurizius, elle aurait pu être la caricature même de la jeunesse instinctive. Or il n'en est rien. À son égard Wassermann n'emploie quasiment pas le terme de « jeune » ou de «jeunesse", si ce n'est a posteriori, alors que, de toutes les femmes du roman, elle est la seule à avoir perdu son éclat avec l'âge. Après dix-huit ans de séparation, Maurizius ne reconnaît plus en elle ce "miracle de la jeunesse» («Wunder der Jugend» p.517) qui en faisait tout le mystère. Si ce personnage est effectivement caricatural, c'est par la représentation de la sensualité et de la beauté féminine réduite à un simple objet de fascination, et non par sa jeunesse. Il est d'ailleurs assez 
caractéristique à cet égard que Max Rychner ne pense pas à Anna Jahn pour illustrer son hymne à la sensibilité juvénile.

Inversement, le personnage du philanthrope américain La Due voit ses qualités humaines clairement associées à la notion de jeunesse: Malgré ses «quarante, quarante cinq ans", il fait partie des hommes-enfants gais, innocents et frais, qui vivent sur la côte pacifique de l'Ouest américain («Im Westen geboren, an der Küste des Pazifik, wo frische, heitere, unbefangene, kindergleiche Männer leben » p. 341).

En mettant en exergue la jeunesse dans sa recension, Max Rychner ne dénature donc pas le roman. Toutefois, sa définition de la jeunesse se distingue de celle de l'article de la Neue Rundschau : il la considère, non comme une période de la vie, mais comme un ensemble de qualités humaines telles que la spontanéité, l'intuition et un instinct dont l'adolescence peut être porteuse, ce que l'on pourrait résumer par le terme de juvénilité.

15 Ce faisant le critique manipule ses lecteurs comme il se fait manipuler par l'écrivain. Car si instinctif soit Etzel, à ce que l'on nous indique à de multiples reprises, ce sont ses qualités de dissimulation, de ruse («List»p.477), de calcul et de réflexion qui lui permettent de mener à bien son projet: retrouver le témoin clé du crime, le faire revenir sur son faux témoignage et laver de tout soupçon le malheureux Maurizius incarcéré à tort depuis dix-huit ans par son père, le procureur général Andergast. Sans être pour autant de fausses pistes, les nombreuses remarques insistant sur la sûreté de l'instinct de Etzel et sur la supériorité du cœur sur l'esprit ne doivent pas aveugler le lecteur, aussi insistantes soient-elles, telle la phrase suivante : «Tandis qu'il cogitait et se torturait l'esprit, son instinct l'avait déjà mis sur la bonne voie » («Während er grübelte und sich den Kopf wund dachte, hatte ihm der Instinkt bereits den richtigen Weg gewiesen » p. 303). L'adolescent est loin d'être un enfant sauvage, sorte d'homme naturel et innocent, vierge de toute culture, poussé uniquement par ses instincts. L'affirmation de Erich Franzen dans la Frankfurter Zeitung est erronée lorsqu'il affirme que «Etzel représente la jeunesse, l'instinct frais et infaillible, par opposition à la correction bornée de son père, l'exprit clair et organique opposé au chaos opaque de Waremme le déraciné » («Etzel vertritt die Jugend, den frischen untrüglichen Instinkt gegen die bornierte Korrektheit des Vaters, den hellen, organischen Geist gegen die undurchsichtige Chaotik des wurzellosen Waremme») $)^{13}$. Etzel Andergast est au contraire un jeune homme raisonné féru de littérature qui peut réciter par cœur des poèmes grecs et latins ou des vers de Rilke et Stefan George pour le plaisir (p. 232-234). En dépit de l'appellation enfantine, le personnage est beaucoup plus riche que ne le laissent supposer les allusions éparses de Victor Zuckerkandl ou Erich Franzen (cf. notes 11 et 13).

16 Si la spontanéité, le sentiment de justice et l'instinct jouent un rôle essentiel, celui-ci se limite à donner une impulsion au protagoniste et à le conforter dans les moments de doute. Sans la perspicacité et l'intelligence qui permettent de tirer profit de ces atouts, Emil Hölcher parle d'une « logique inébranlable» (« unerschütterliche Logik», cf. note 5), aucune action efficace n'est envisageable. Les qualités de stratège du jeune homme sont d'ailleurs soulignées dés le début: «Il constatait une fois de plus qu'il était impossible d'obtenir quelque chose de quelque personne que ce soit, fût-elle la plus insignifiante, en allant droit au but, il fallait duper l'autre et le mystifier sur ce que l'on voulait en tirer, cela revenait toujours à tendre un piège » («Er sah nur wieder einmal, $\mathrm{da}$ auf geradem Weg von keinem Menschen was zu erreichen war, auch vom 
harmlosesten nicht, man mußte jeden überlisten und über das, was man von ihm haben wollte, hinters Licht führen, es war immer eine Fallenstellerei » p. 88). Il n'hésitera d'ailleurs pas à mettre ces qualités en pratique de nombreuses fois pour démasquer Waremme-Warschauer, la plus flagrante étant la simulation d'une grave maladie pour extorquer un récit circonstancié du meurtre dont est accusé Maurizius ( Was an leidenschaftlicher List, an andergastscher Hartnäckigkeit, an sechzehnjährigem Feuer in diesem Kopf und Gemüt aufgespeichert war, wirkte sich nun dämonisch in der Vorbereitung auf die entscheidende Stunde aus » p. 477). Or ces traits de caractère que sont la ruse, la patience, la perspicacité et l'intelligence ne sont pas l'apanage d'un âge plus que d'un autre. De même que les générations précédentes ne sont pas exemptes de sensibilité et d'instinct comme le montre bien Wassermann. L'insistance de la Literarische Welt sur la jeunesse n'en est que plus problématique.

De cette confrontation entre le roman der Wassermann et la recension de Max Rychner renforcée par la comparaison entre la critique publiée par la Literarische Welt et les autres jaillissent un certain nombre de questions :

Pourquoi la Literarische Welt distingue-t-elle plus particulièrement l'adolescence de l'enfance ? Pourquoi accorde-t-elle plus d'importance à la notion de jeunesse qu'à la justice et qu'au judaïsme dans ce roman? Pourquoi donne-t-elle une vision aussi idéalisée de la jeunesse? Pourquoi fait-elle du jeune Etzel Andergast le symbole d'une génération?

Le regard si subjectif du commentateur ne serait-il pas influencé par l'intérêt particulier que portent les rédacteurs de la Literarische Welt à la jeunesse ? C'est ce que j'essaierai de montrer dans cet article.

Il est fort à parier que Max Rychner s'est laissé entraîner par les déclarations de Jakob Wassermann dans le périodique littéraire et par l'intérêt qu'il affichait pour la jeunesse intellectuelle. En effet, le 14 janvier 1927, la revue publiait un texte de l'écrivain consacré à la jeunesse. Il s'agissait d'une série de cinq lettres ouvertes intitulées "Worte an die Jugend" publiées en première page, dans laquelle des auteurs renommés tels que Thomas Mann («Worte an die Jugend (1)», in $L W$, Nr. 1, 1927 p. 1), René Schickele ("Junge Dichter in Deutschland», in $L W$, Nr. 3, 1927, p. 1) et Heinrich Mann ("Die neun Gebote», in $L W$ Nr. 3, 1927, p. 1) acceptaient de s'adresser à la jeunesse. Dans son texte, publié en second ${ }^{14}$, Jakob Wassermann affirme à quel point "la question éternelle, la question actuelle par excellence» («Die ewige, die heutige Frage aller Fragen »), la jeunesse donc, le passionne jusqu'à être au cœur même du livre qu'il rédige à cette époque. Or, si l'on en croit la chronologie, il s'agit très certainement de L'affaire Maurizius.

Lieber Herr Haas, die Aufforderung, die Sie an mich richten, ich möge an die geistig produktive Jugend Deutschlands ermunternde Worte richten, hat mich tagelang beschäftigt und die widerstreitendsten Gedanken und Gefühe in mir erregt, um so mehr als das Werk, an dem ich nun seit vielen Monaten arbeite und zu dem frühere Bücher gleichsam nur Vorspiele waren, sich in Gestaltungen bewegt, deren brennender Kern eben dieses ewige Problem ist: Jugend. Die ewige, die heutige Frage aller Fragen.

Cher Monsieur Haas, la demande que vous m'avez faite d'adresser quelques mots d'encouragement aux jeunes créateurs allemands d'œuvres intellectuelles m'a occupé des jours entiers et a suscité en moi les sentiments et les idées les plus contradictoires, et ce d'autant plus que l'œuvre à laquelle je travaille maintenant depuis des mois, et en comparaison de laquelle mes livres précédents ne constituaient qu'un prélude, s'élabore justement autour du sujet brûlant qu'est ce 
problème éternel: la jeunesse. Cette question éternelle, question actuelle par excellence.

21 dection de Willy Haas, se donna pour mission de soutenir et din " jeunesse ». Toutefois en dépit d'une formulation à caractère universel, la « jeunesse " de la Literarische Welt ne représente qu'une minorité. Il s'agit uniquement des jeunes lecteurs potentiels de la revue, dont on peut supposer que la majorité étaient de simples amateurs de littérature, auxquels viennent s'ajouter les jeunes écrivains allemands "die geistig produktive Jugend Deutschlands", comme l'écrit Jakob Wassermann en janvier 1927. Sur ce point l'écrivain et la revue se rejoignent, car le jeune Etzel Andergast fait des études et se passionne pour la littérature et la philosophie incarnées dans le roman par Melchior Ghisels. C'est ce qui fait réagir les commentateurs marxistes contre cette "fuite dans la littérature $»^{15}$ quelques années plus tard, tandis que l'écrivain s'adresse de nouveau à la jeunesse estudiantine, cette fois dans la Neue Rundschau ${ }^{16}$. La " jeunesse ", "problème éternel » est donc à prendre dans un sens restrictif. Elle se limite à celle à laquelle Wassermann veut pouvoir s'identifier en devenant son porte-parole: Des jeunes gens de «quatorze, seize, dixneuf ans ", comme il l'indique dans l'article de janvier 1927, déçus par une société en crise qui les condamne en outre à l'anonymat. Mais dans le portrait qu'il en dresse, celui de jeunes gens qui ne se laissent embrigader par "aucune confrérie, aucun groupement, aucune association, aucun groupe politique ou groupe de pression» ( «Sie, an die ich denke, gehören keinen Genossenschaften, Bünden und Vereinigungen an, keinen politisierenden und Ertüchtigungsmannschaften; es sind einzelne, [...] verstreut in allen Städten und Provinzen des Landes », in "Worte an die Jugend », $L W$ Nr. 2, 1927 p. 1), relève beaucoup plus de l'idéal que de la réalité. On y retrouve l'incorruptible Etzel Andergast, représentant de ces jeunes cœurs purs passionnés de littérature qu'imaginent les rédacteurs de la Literarische Welt dans les premières années de parution par opposition à la génération d'avant-guerre et à son "sens du devoir » déshumanisé dont le procureur général Andergast est le meilleur représentant dans L'affaire Maurizius.

En dépit des apparences, cette attitude de Jakob Wassermann n'est pas totalement dénuée d'un certain réalisme dans la mesure où l'écrivain préfère s'exprimer sur ce qu'il connaît le mieux et ne prétend pas représenter toute la jeunesse, tout comme la rédaction de la Literarische Welt a conscience de ne s'adresser qu'à une faible part de la population. Leurs remarques sont bien plutôt le fruit d'une quête : celle de jeunes gens responsables et créatifs, voire de futurs écrivains, capables de donner un nouvel essor à la culture et à la démocratie allemande ${ }^{17}$. Cette recherche les conduit assez naturellement à mettre l'accent sur deux points essentiels que sont la différence marquée entre la jeune génération et celle qui la précédait, d'une part, ce qui se traduit dans L'affaire Maurizius par un conflit de générations assez classique, et le refus de la contemplation, d'autre part. De ce point de vue, les étudiants marxistes de Student im Kampf se fourvoient lorsqu'ils interprètent la mise en exergue d'une jeunesse férue de littérature par Jakob Wassermann, et donc par la Literarische Welt, comme une propension à la réclusion volontaire dans un monde fictif à l'écart du monde réel (cf. note 15). Max Rychner salue au contraire l'esprit d'initiative de Etzel Andergast et sa capacité à mettre le verbe en action: «Etzel ist kein jungradikaler Literat, dem 
'Verwirklichen'eine bloße drohende Vokabel bleibt, er ist ein Täter des Worts. Und er gelangt zum Ziel ». Etzel n'est donc pas qu'un adorateur du verbe, il est celui qui le met en pratique.

Par cette remarque, Max Rychner laisse sourdre l'une des angoisses de Willy Haas, le rédacteur en chef de la Literarische Welt, et de nombre des autres rédacteurs. En effet, les étudiants marxistes ne sont pas les seuls à condamner la tendance d'une partie de la jeunesse intellectuelle à s'abstraire du monde réel, et à s'en inquiéter. Dans une interview de Heinrich Mann sur divers sujets d'actualitée ${ }^{18}$, Willy Haas relativise l'optimisme de l'écrivain :

Ich bin nicht so optimistisch wie Sie. Mich erschrecken vor allem die Zwanzigjährigen mit ihrer furchtbaren Indifferenz. Gerade deshalb, weil wir Dreißig- bis Fünfunddreißigjährigen einmal - vor 1914 - ebenso indifferent waren wie jene. Sie, Herr Mann, haben als Erster gewarnt und ermahnt, - lange vor 1914. Aber die meisten haben Ihnen nicht geglaubt. Erst mußte der Krieg kommen...

[...] Wir, die den Krieg im Schützengraben mitgemacht haben, sind tief erbittert darüber, daß unter unseren jüngeren Kameraden alles wieder so wird wie vor 1914: Artistik, luxuriöse und komplizierte Psychologie... und gar kein Blick für die wirklichen bewegenden Kräfte unserer Zeit.

Je ne suis pas aussi optimiste que vous. Je suis surtout effrayé par les jeunes de vingt ans et leur effroyable indifférence. Et ce justement parce que nous, hommes de trente à trente cinq ans, étions, avant 1914, tout aussi indifférents qu'eux. Vous, Monsieur Mann, avez été l'un des premiers à mettre en garde et à menacer, bien avant 1914. Mais la plupart ne vous ont pas cru. Il fallait que la guerre arrivât...

Nous qui avons fait la guerre dans les tranchées sommes profondément amers de voir que chez nos jeunes camarades tout recommence comme avant 1914: l'art pour l'art, la psychologie compliquées et fastueuse et pas un regard pour les véritables forces de mouvement de notre temps.

Certes la contemplation ne touche qu'une minorité parmi la jeunesse intellectuelle, elle-même minoritaire, et elle ne peut pas être considérée comme un signe des temps, puisqu'elle existait déjà avant 1914 comme le remarque Willy Haas. Toutefois, dans un contexte politique où la démocratie n'est pas encore devenue une habitude, pour paraphraser Heinrich Mann dans le même article («Machet die Republik zur Gewohnheit »), «l'indifférence » peut s'avérer tragique. C'est pourquoi les rédacteurs de la Literarische Welt pourfendent cette attitude au fil des années en même temps qu'ils poursuivent leur tentative de cerner la jeunesse allemande et ses difficultés, afin d'y remédier.

C'est donc fondamentalement à l'émergence d'une jeunesse nouvelle et d'une nouvelle perception de cet âge par les jeunes eux-mêmes que se consacre la Literarische Welt, car, écrit Jakob Wassermann en janvier 1927 : «Dans les années 1880-1890, être jeune ne signifiait rien de particulier. On ne pouvait pas se prévaloir d'être jeune, on n'avait même pas le droit de s'en réclamer, et, comme en témoigne ma douloureuse expérience, le soin et la considération dont on pouvait jouir de ce fait étaient nuls » ( «In den Jahren 1880 bis 1900 was es nichts Bemerkenswertes, jung zu sein. Man konnte nicht darauf pochen, daß man jung war, man durfte sich nicht einmal darauf berufen, und die Obsorge und die Beachtung, die man deswegen genoß, ich kann da aus eigener schmerzlicher Erfahrung sprechen, war gleich null». (cf. note 14).

Presque cinquante ans après la publication des Frères Karamasov, en 1928 le conflit pèrefils est loin d'être une innovation de la littérature européenne. On en trouve quelques exemples dans les commentaires critiques des dernières parutions de la Literarische 
Welt. Toutefois, «l'épouvantable incompréhension entre les générations, qui fait que les plus anciens croient les plus jeunes a priori imperméables aux souffrances qu'ils ont, pour leur part, endurées dans le passé et auxquelles ils sont devenus trop sourds avec le temps", est considéré par la Literarische Welt comme un fait unanimement constaté (cf. Jean R. Kuckenburg: «Buch-Chronik: Marie Hamsun: Die Langerundskinder", in LW, 1928, Nr. 2, p. 6: «[...] die grauenhafte Entfremdung zwischen den Generationen, daß die Älteren glauben, die Jüngeren seien gegen die Leiden von vornherein gefeit, die sie selbst früher schmerzhaft durchlebt haben und für die sie mit der Zeit zu stumpf geworden sind»). Cette récurrence de l'incompréhension entre les générations au fil des articles (cf. «Zuschriften: Aufruf an die Jugend », in $L W, 1928$, Nr. 6, p. 9, Ernst Glaeser : "Das Märchen von der Freiheit », in $L W, 1931, \mathrm{Nr} .51, \mathrm{p} .3 / 4)$ prouve à quel point ce problème préoccupe les rédacteurs, d'autant qu'une simple différence d'âge de 10 ans semble rédhibitoire (-o-: «Glossarium », in $L W, 1928$, Nr. 8, p. 4). Il est vrai qu'en 1928, les hommes de trente ans avaient vécu la Première Guerre mondiale, ce qui creusait un fossé infranchissable entre eux et leurs cadets âgés de vingt ans. C'est d'ailleurs la guerre qui marque la frontière entre la jeunesse et le reste de la population puisqu'en 1931 Emil Belzner définit comme jeunes les individus âgés de 20 à 30 ans $^{19}$.

L'expression "conflit père-fils", avec toutes ses implications psychanalytiques, est donc inappropriée. En dépit des apparences initiales, c'est bien plutôt d'incommunicabilité entre les générations qu'il faudrait parler pour rendre compte à la fois du roman de Wassermann et des préoccupations de la Literarische Welt.

Il est clair que le jeune Etzel n'agit pas pour ridiculiser son père mais parce qu'il a une conception de la vie radicalement différente et veut défendre ses idéaux à tout prix. Pour l'essentiel les deux personnages sont d'ailleurs séparés par des centaines de kilomètres, le père à Francfort, le fils à Berlin. Néanmoins, même sans conflit ouvert, l'opposition entre deux générations que relève Max Rychner ( $Z$ Zwei Generationen stehen gegeneinander, zwei Welten ») est bien réelle. Ce faisant, le baron («Freiherr ») Wolf von Andergast, procureur général de son état, incarne non seulement une génération, mais une catégorie bien connue de la société allemande que dénonce Wassermann comme l'une des plaies de la République, ce qui explique le caractère caricatural du personnage : Andergast est l'un de ces fonctionnaires zélés de l'Ancien Régime pétris d'un «sens du devoir» tout prussien, qui vise à faire oublier la complexité de l'âme humaine, à commencer par leur propre sensibilité. Le procureur Andergast, car jamais, même dans ses relations avec son fils il ne se départ de sa dignité professionnelle, n'est pas un « monstre », mais ce n'est plus un « homme» :

Kann jedoch die formale Logik des Staatsanwaltes Andergast den Fall warhrhaft erkennen, den die untergründig unlogischen Elementar-mächte der Menschenseele geschaffen haben? Andergast ist kein Unmensch, aber er ist kein Mensch, er ermangelt der Intuition des Herzens, die ihn die Augenscheinlichkeiten seines Verstandes zur Ahnung wahrerer Wirklichkeiten erhöhe und zum fruchtbaren Zweifel an der eignen Unfehlbarkeit. (Max Rychner, cf. note 9)

Ce peut-il que la logique formelle du procureur Andergast soit en mesure d'appréhender véritablement ce dossier, que les forces élémentaires sous-jacentes et illogiques de l'âme humaine ont crée ? Andergast n'est pas un monstre mais ce n'est pas un homme, il lui manque l'intuition du cœur qui transformerait l'évidence de son intellect en intuition des véritables réalités en un doute fructueux sur sa propre infaillibilité. 
Que ce soit sa mère, qui appréhende toujours ses visites, bien qu'elle soit de taille à lui répliquer, son ex-femme, qui ne se laisse plus intimider, sa maîtresse ou son fils, Andergast les traite tous avec une distance et une froideur propre à l'administration du II ${ }^{e}$ Reich jusqu'à ce que Etzel lui échappe et que le doute s'immisce en lui. Il est dès lors perdu, car cet homme de l'ordre n'est pas préparé à une telle rencontre avec l'irrationnel: le doute le ronge jusqu'à le précipiter dans la folie. Face à cet excès de déshumanisation dans un premier temps puis d'irrationalité dans un second, le jeune Etzel incarne un juste équilibre. Par delà les apparences d'un conflit de générations bien ordinaire, Jakob Wassermann exprime son pessimisme quant aux facultés d'adaptation de ses concitoyens d'âge mûr. En effet, à la banalité de la situation s'ajoute la situation spécifique de l'Allemagne pour laquelle le changement de génération va de pair à cette époque avec le traumatisme de la Première Guerre mondiale, la défaite, l'effondrement du IIe Reich et la fondation de la République de Weimar, autant d'événements qui ne peuvent que renforcer la différence de perception du monde des générations successives. Non seulement leur regard, mais le monde qu'ils observent ont changé. La transition vers la République n'est pas seulement d'ordre idéologique, elle nécessite un changement de comportement que Wassermann ne croit pas envisageable. Ce pessimisme ne peut dès lors plus être contrebalancé que par la foi en la jeunesse, appelée à remplacer ses aînés à plus ou moins court terme.

31 Autant le procureur Andergast constitue un pôle de répulsion, autant son fils Etzel sert, pour sa part, de pôle d'attraction. Il incarne avant tout « l'idéal d'un jeune de notre époque obsédé par la volonté d'une justice sans faille » que Jakob Wassermann voudrait donner à ses jeunes concitoyens comme l'écrit avec beaucoup de clairvoyance le critique Kurt Martens («sein sechzehnjähriger Sohn, das Ideal von einem Jungen unserer Zeit, besessen vom Willen zur schlackenreinen Gerechtigkeit », cf. note 6). Erwin Poeschel n'écrit pas autre chose dans le quotidien suisse Der Bund (Erwin Poeschel : «'Der Fall Maurizius'. Der neue Roman von Jakob Wassermann », in Der kleine Bund. Beil. zu Der Bund (Bern) Jg. 9, Nr. 10, 4. März 1928, p. 78-80).

Ce qui fait du jeune Etzel Andergast un homme idéal n'est autre que la symbiose entre des qualités d'ordre totalement irrationnel (intuition du cœur, instinct, flamme) et une parfaite maîtrise de soi grâce à l'intelligence et au raisonnement, qualités rationnelles par excellence. Il faut toutefois noter que «l'instinct» tant vanté par l'auteur, ne s'apparente en aucune façon à une quelconque forme de bestialité, il s'agit en l'occurrence de l'instinct $\mathrm{du}$ bien, d'une attraction naturelle vers la justice ( Gerechtigkeit »). Cette définition de « l'instinct » chez Etzel est explicitement donnée par son professeur Camill Raff, qui paiera d'ailleurs fort cher sa sympathie pour le jeune homme :

[...] er habe niemals Verschlagenheit an Etzel wahrgenommen, etwas anderes wohl, einen auffallenden Scharfsinn oder Spürsinn, das wohl, eine Art Indianerinstinkt, wenn es gilt, verborgene Dinge oder Umstände ans Licht zu bringen. (p. 130)

Il n'avait jamais remarqué de rouerie chez Etzel, il s'agissait d'autre chose: d'une lucidité ou d'une intuition étonnantes, oui c'était cela, une sorte d'instinct de Sioux, si c'est possible, pour mettre en lumière les circonstances ou les choses cachées. fasse plutôt preuve d'une hypersensibilité pour les nobles causes. Le caractère instinctif de ce comportement tient plus à ses aptitudes de pisteur infatigable, représentées par le chasseur indien, qu'à une animalité quelle qu'elle soit. Il est d'ailleurs tout à fait évident dans le texte que cet « instinct » s'est développé au contact de la littérature, et 
plus particulièrement par le biais des textes du fictif Melchior Ghisels. Même si l'on n'exclut pas la possibilité que le jeune Etzel aient des prédispositions, un "bon flair » («Witterung», cf. note 13), il est manifeste qu'elles se sont développées essentiellement au contact des livres. Ils constituent d'ailleurs une grande part du son bagage (p. 138), alors que son sac à dos, plein à craquer, est si lourd qu'il peut à peine le lever de terre (p.113). Jakob Wassermann insiste à plusieurs reprises sur cet aspect essentiel du jeune personnage. Si la littérature ne signifie pas pour lui un verbe mort, c'est parce qu'elle est aussi incarnée par des contemporains réels (Stefan George, Rilke) ou fictifs (Melchior Ghisels). Au plus fort de sa détresse, alors qu'il désespère d'obtenir de Waremme-Warschauer les informations pour lesquelles il s'est donné tant de peine, l'adolescent se rend chez l'écrivain qu'il vénère le plus :

Um nicht anmaßend zu erscheinen, läßt er nur durchblicken, daß seine Stellung zu prinzipiellen Lebensfragen von den Schriften Ghisels'entscheidend beeinflußt worden ist. Er hat sich jedoch nicht mit der Reflexion begnügt, er ist einen Schritt weiter gegangen. Diesen Sinn hat er eben darin entdeckt : daß man einen Schritt weiter gehen muß. (Melchior Ghisels wird sichtlich aufmerksamer). (p. 362)

Afin de ne pas sembler présomptueux, il laisse uniquement transparaître que son attitude face aux questions essentielles de la vie a été influencée de façon déterminante pas les écrits de Ghisel. Il ne s'est toutefois pas contenté de la réflexion, il est allé au-delà. Il y avait justement découvert cette signification : qu'il faut aller au-delà. (Melchior Ghisel devient visiblement plus attentif).

L'homme de lettres ne donne aucun conseil au jeune homme, mais comme l'indique le personnage lui-même, Ghisels lui avait permis de découvrir par son œuvre « qu'il faut toujours aller plus loin » et il avait déjà franchi ce pas. Il n'a donc plus besoin de tutelle. La littérature lui a donné les outils nécessaires pour devenir un sujet autonome. Il peut désormais se fier à son "instinct». Ce serait donc faire fausse route que de voir dans cette valorisation de l'instinct uniquement un écho du vitalisme. Nous sommes très loin de la force originelle nietzschéenne. Etzel Andergast est un jeune lettré idéaliste, ce sont des valeurs humanistes classiques, dont le sens de la justice, qui le conditionnent. Son « instinct » ne s'oppose pas à son érudition, il en est le fruit.

Cette perception de «l'instinct » est en parfaite adéquation avec le discours diffusé par la Literarische Welt. En effet, malgré une différence apparente, s'agissant de la définition de la jeunesse, l'écrivain et le périodique défendent la même conception.

Certes, l'ouvrage de Wassermann a un contenu politique indéniable que la Weltbühne et la Christliche Welt ont immédiatement décelé et mis en valeur. L'affaire Maurizius est un plaidoyer contre les peines de prison à vie. Wassermann ne cache d'ailleurs pas son intention. La fin de la seconde partie consacrée à l'annonce de sa prochaine libération au prisonnier Maurizius (p.434-464) n'est ni plus ni moins qu'un manifeste contre ce type de peines. Des personnages comme le gardien de prison Klakusch sont là pour prouver que le visage de la justice ne doit pas nécessairement être inhumain (p. 455-461.). Cependant, ce contenu politique relève plus de l'esprit civique que d'une réelle politisation. Les remarques de l'auteur sur la République de Weimar et ses dysfonctionnements (p. 364-365) ne mettent en cause aucun parti politique spécifique mais plutôt un sentiment général de crise («Ich verstehe, Sie sprechen von Politik als von der sozialen Politik überhaupt » p. 364). Son appel à la jeunesse s'inscrit donc dans un cadre plus abstrait qui est celui de la Literarische Welt.

On pourra reprocher à cette attitude son inconscience face à une situation d'urgence. Jakob Wassermann décrit lui-même l'abîme vers lequel se précipite l'Allemagne : 
[...] heute bedroht die Erschütterung das ganze Gebäude, das wir seit zweitausend Jahren aufgerichtet haben. Es regt sich eine tiefe, kranke Zerstörungslust in den empfindlichsten Teilen der Menschheit. Wenn dem nicht gesteuert werden kann, und ich fürchte, es ist zu spät, muß in den nächsten fünfzig Jahren zu einem ganz furchtbaren Zusammenbruch kommen, weit über die bisherigen Kriege und Revolutionen hinaus (p. 364)

[...] aujourd'hui l'édifice tout entier que nous avons construit depuis deux mille ans menace de s'écrouler. Une volonté de destruction maladive, profonde, se fait jour là où l'humanité est la plus sensible. Si l'on ne peut pas la guider, et je crains qu'il ne soit trop tard, elle conduira irrémédiablement dans les cinquante prochaines années à un effondrement tout à fait effroyable, bien au-delà des guerres et des révolutions connues jusqu'ici.

Mais au lieu de réagir par une mise en cause directe des dangers politiques immédiats que représente la montée du nationalisme, il choisit une option à très long terme dont les chances d'aboutir sont hypothétiques: la formation intellectuelle de la jeunesse, dont seule une minorité semble réceptive à ces solutions. Peut-être ce manque de combativité s'explique-t-il par la perspective adoptée : à l'échelle de l'humanité et non de l'Allemagne.

Quoique les rédacteurs de la Literarische Welt adhèrent à l'idée selon laquelle seule une éducation solide de la jeunesse pourrait redresser la situation, ils sont conscients de ses limites dans une situation d'urgence. C'est pourquoi on peut lire simultanément dans le périodique des articles qui réitèrent régulièrement la nécessité de développer une conscience politique démocratique, la culture et le libre arbitre de la jeunesse, et des cris d'alarmes face à l'absence de valeurs de la jeune génération, car, tous en ont conscience, Etzel Andergast n'est pas représentatif de la jeunesse réelle de ce pays. Heinrich Mann est à cet égard l'un des auteurs les plus prolixes de la Literarische Welt dont les articles suscitent souvent un débat. C'est par leur optimisme que se distinguent les interventions de l'écrivain lubeckois. Dans un article intitulé « Früher und jetzt $»^{20}$ publié en page de garde du numéro anniversaire des dix ans de la République de Weimar, Heinrich Mann dresse un portrait effrayant de la jeunesse contemporaine sans pour autant en tirer de conclusions négatives. D'autres rédacteurs s'en chargent à sa place. Cette nouvelle jeunesse qui se caractérise avant tout par son sentiment absolu d'immédiateté ( « Gegenwärtigkeit »), explique-t-il, n’a peur de rien : « Gegenwärtig sein gilt als Tugend. Es ist auch wirklich eine, denn so bleibt man furchtlos. Das wichtigste einer solchen Jugend ist ihre Furchtlosigkeit ». À cela s'ajoute une absence complète de sens historique, à croire que ces jeunes sont «tombés du ciel». Leur horizon, passé ou futur, ne dépasse pas six mois : "Vor allem sind sie unhistorisch, als wären sie vom Himmel gefallen. Vor sechs Monaten war, wenn man sie hört, noch keiner da, und wer sie in sechs Monaten sein werden, findet sich». Cette soif de vivre intensément l'instant au détriment de toute autre dimension temporelle, associée à une indéniable violence née dans les tranchées et renforcée par la peur du lendemain lors de la grande inflation, engendre une remise en question de la morale traditionnelle. C'est pourtant sur une note positive que se termine l'article : ces jeunes qui ont su se créer une morale nouvelle sont, affirme l'écrivain, soudés par un fort sentiment communautaire à tous les niveaux, y compris celui de la création artistique.

Dans sa critique du roman de Frank Thieß Der Zentaur, consacré à la jeunesse, Emil Belzner tire des conclusions beaucoup plus noires d'un exposé analogue sur la jeunesse (cf. note 19). Mais il est vrai que son article paraît en juillet 1931 à une époque déjà plus trouble de la République de Weimar. Contrairement à Heinrich Mann qui justifie le 
refus des valeurs humanistes de la jeunesse par leurs difficultés économiques (cf. notes 18 et 20), il émet un tout autre diagnostique. L'errance de la jeunesse écartelée entre des domaines aussi hétéroclites que "la culture, la technique, le christianisme, le paganisme, le pacifisme, le patriotisme, le sentimentalisme, la sportivité, l'idée et l'arbitraire», véritable «tohu-bohu» («Tohuwabohu»), vient à son avis de leur méconnaissance de la nature même de la culture et du rejet qui en découle.

Or, écrit Emil Belzner, en conclusion de son long article, la culture n'est rien d'autre qu'une mise en pratique ("Verwirklichung»). Toute la question est de savoir si la jeunesse sera capable de le comprendre et de vivre ce dont elle n'a qu'une approche théorique. Car seule une culture vécue, expérimentée, permet de mettre en adéquation la réflexion et l'action écrit-il en conclusion. Ce faisant il reprend ce qu'il affirmait trois colonnes auparavant :

Eine neue Jugend und mit ihr eine neue Menschheit wird erst dann erstehen, wenn sie die Verpflichtungen einsieht und anerkennt, die ihr die bisherigen Leistungen des menschlichen Herzens und des menschlichen Verstandes auferlegen. Kultur als schöner Selbstzweck, als Ferienaufenthalt im Dasein, ist ein Irrtum; Kultur als Forderung, als großartige Anleitung zur Verwirklichung, ist eine Wahrheit, eine Wahrheit, die es Tag für Tag zu mehren und zu befolgen gilt. Eine Aufgabe der Jugend, dafür $\mathrm{zu}$ sorgen, daß sich allmählich Kultur und Wirklichkeit zusammenwachsen, nicht gegeneinander. Eine Definition des nicht gerade eindeutigen Begriffs "Kultur « wäre hier am Platze. Ich meine damit das Suchen und Tasten nach dem Menschenwürdigen. Zwischen ihm und dem geringsten Menschenleben muß der Zusammenhang hergestellt werden.

Une nouvelle jeunesse et, avec elle, une nouvelle humanité ne naîtra que lorsqu'elle aura admis et reconnu les devoirs que lui imposent les performances accomplies jusqu'alors par le cœur et l'intelligence humaine. C'est une erreur de considérer la culture humaine comme un joli but en soi, comme un lieu de villégiature de l'existence. La culture en tant qu'exigence, en tant qu'indication grandiose en vue de la réalisation est une vérité, une vérité qu'il faut augmenter et suivre jour pour jour. C'est une mission de la jeunesse de veiller à ce que, peu à peu, culture et réalité se fondent au lieu de s'opposer. Une définition du concept somme toute peu explicite de la " culture » s'imposerait ici. J'entends par-là le tâtonnement vers ce qui est digne de l'homme. C'est entre cette dignité et la vie humaine la plus médiocre que doit être établi un lien.

C'est l'avenir même de la société, et non seulement de la jeunesse, qui est en jeu, explique Emil Belzner.

À la lecture de ces remarques, la parenté de ce discours avec les commentaires de Max Rychner sur le personnage de Etzel Andergast et sur sa mise en pratique du verbe s'impose: «Etzel ist kein jungradikaler Literat, dem 'Verwirklichen'eine bloße drohende Vokabel bleibt, er ist ein Täter des Worts ", écrivait-il trois ans et demi plus tôt. Mais le simple fait que cette symbiose entre la culture et l'action de l'individu ne soit toujours pas réalité dans la jeune génération en 1931 ne prouve-t-il pas que le personnage de Etzel n'est qu'un idéal à poursuivre, idéal d'autant plus improbable que la bourgeoisie, classe sociale traditionnellement porteuse de la culture, a été fortement ébranlée par les crises économiques successives, comme l'indique d'ailleurs assez bien Heinrich Mann dans son article «Früher und jetzt » (cf. note 19) ? Cependant toutes ces recherches apparemment infructueuses pour définir la jeunesse de la République de Weimar occulte une autre question :

En recherchant une jeunesse idéale, ces hommes ne courent-ils pas après une symbiose entre la culture et l'action humaine qui n'aurait jamais existé? 


\section{NOTES}

1. Stephen H. Garrin : The Concept of Justice in Jakob Wassermanns Trilogg, Peter Lang, Bern, 1979.

2. Engel Fritz : «'Der Fall Maurizius'. Ein Buch der Zeit zur rechten Stunde », in Berliner Tageblatt, 22. Feb. 1928 Nr.89, 1. Beiblatt et Korrodi Eduard: "Der Fall Maurizius. Bemerkungen zu Wassermanns Roman », in Neue Zürcher Zeitung, 28. Feb. 1928, Nr.366, Blatt 5.

3. Weltsch F. : «Wassermanns Kampf mit dem Judentum », in Jüdische Rundschau, Berlin, 1928, p. 554-556.

4. Pätzold Erwin: «Der Dichter vor dem Richter. Zu Jakob Wassermanns Roman 'Der Fall Maurizius' », in Eckart, Juli/Aug., 1928, Jg. 4, H. 7/8, Berlin, p. 312-314.

5. Hölcher Emil : «Der Fall Maurizius », in Die Christliche Welt, 18. Aug. 1928, Jg. 42, Nr.16, Gotha, p. 772-776.

6. Martens Kurt: «Der Fall Maurizius », in Die Literatur, 30. Jg. 1927-1928 p. 544 et Einsiedel Wolfgang von : "Nur ein Roman. Zu Wassermanns 'Fall Maurizius' », in Die schöne Literatur, Okt. 1928, Heft 10, p. 478-481.

7. Certes, l'article de H. Rémy défend aussi ce point de vue, mais l'antisémitisme primaire de ce discours relève bien plus de la propagande nazie la plus grossière que de la critique littéraire : Univ.-Prof. $\mathrm{D}^{\mathrm{r}} \mathrm{H}$. Remy: «Deutschlands bester Gegenwartsroman? Zu Jakob Wassermanns neuestem Werk 'Der Fall Maurizius'», in Schönere Zukunft, 10. Juni 1928, Jg. 3, Nr. 37, Wien, p. 802-803.

8. Regensteiner Henry: "The status of Education in the Light of Jakob Wassermann ", in Revue des langues vivantes, Liège, 1964, p. 621-623 et Oonk Mariana M. : Jakob Wassermanns Auffassung des "neuen Menschen », Diss. Abstracts International Ann Arbor, 1971, (U. of Wash).

9. Rychner Max: «Wassermanns neuer Roman 'Der Fall Maurizius'», in Die literatische Welt, Berlin, 2. März 1928, Nr.9, p. 5.

10. Les numéros de pages indiqués entre parenthèses renvoient à l'édition suivante : wassermann Jakob : Der Fall Maurizius, dtv, München, 2000.

11. Zuckerkandl Viktor : «Der Fall Maurizius », in Die Neue Rundschau, 1928, Berlin, p. 426-434.

12. Une recherche sur le CD-Rom « Deutsche Literatur von Lessing bis Kafka » Digitale Bibliothek 1 , montre que chez aucun des auteurs traités, le terme "jugendlich» n'est utilisé dans son acception moderne d'adolescent. Le synonyme «der Halbwüchsige » n'est pas plus utilisé, à croire que jusqu'à une période récente, la littérature ne distinguait pas l'adolescence de l'enfance.

13. Erich Franzen: «Maurizius oder: Unschuld und Sühne». $\mathrm{Zu}$ dem Roman von Jakob Wassermann, in Frankfurter Zeitung, 1. April 1928, Literaturblatt Nr.14.

14. Wassermann Jakob : «Worte an die Jugend (2)», in Die literatische Welt, 1927, Nr. 2, p. 1.

15. Strzelewicz Willy: "Die Flucht in die Literatur, Gedanken zu Wassermanns 'Der Fall Maurizius' », in Student im Klassenkampf, 1933, H. 5, Wien.

16. Wassermann Jakob: "Rede an die studentische Jugend", in Die Neue Rundschau 1932, p. 529-546.

17. Pour une présentation générale de la jeunesse dans la Literatische Welt, cf. Avenel-Cohen Pascale : Willy Haas et le périodique «Die Literarische Welt "1925-1938, Presses du Septentrion, Lille, 1997, p. 331-352, ici p. 335.

18. «Zeitprobleme/Gespräch mit Heinrich Mann », in Die literatische Welt, 1927, Nr. 44, p. 1.

19. Emil Belzner, «Wohin treibt die Jugend? », in Die Literarische Welt, 1931, Nr. 27/28 p. 1/2.

20. Heinrich Mann : «Früher und jetzt », in Die Literarische Welt, 1928 Nr. 45, p. 1. 


\section{RÉSUMÉS}

Dès sa parution en février 1928, le roman de Jakob Wassermann L'affaire Maurizius suscita un intérêt non négligeable parmi les critiques littéraires et les hommes de lois. Parallèlement à la critique de l'appareil judiciaire de la République de Weimar et à une interrogation fondamentale sur la justice, le judaïsme et l'assimilation constituent le second pôle développé par la presse et les commentateurs non seulement à l'époque mais aussi après la guerre. Pourtant, certaines de ces recensions focalisent leur analyse sur une question apparemment secondaire: la représentation de la jeunesse par le biais du personnage de Etzel Andergast. C'est sur cette focalisation que porte le présent article. Il s'interroge sur les raisons de la mise en exergue de la jeunesse ainsi que sur l'image de cette génération véhiculée par Jakob Wassermann, par ce roman en particulier et par des périodiques tels que Die Literarische Welt ou Die Neue Rundschau en les confrontant aux autres articles parus à l'époque.

Schon bei seiner Veröffentlichung im Februar 1928 erregte Jakob Wassermanns Roman Der Fall Maurizius ein beträchliches Aufsehen unter den Kritikern der literarischen Presse und den Juristen. Parallel zu einer Kritik der Justiz der Weimarer Republik und zu einer gründlichen Reflexion über die Gerechtigkeit bilden Judentum und Assimilation den zweiten Pol, mit dem sich die Presse und die Kritiker damals wie auch nach dem zweiten Weltkrieg befaßten. Jedoch fokussieren manche Rezensionen ihre Analyse auf einen scheinbar nebensächlichen Aspekt, nämlich die Darstellung der Jugend durch die Figur des Etzel Andergast. Eben diese Fokussierung liegt vorliegendem Artikel zugrunde. Es gilt, sich zu fragen, warum Jakob Wassermann, unter anderem in diesem Roman, und manche literarische Zeitschriften wie Die literarische Welt und Die neue Rundschau den Akzent auf die Jugend und die Darstellung der damaligen jungen Generation setzten. Ein Vergleich mit den anderen damaligen Rezensionen soll dazu beitragen, eine Antwort zu finden.

\section{AUTEUR}

\section{PASCALE AVENEL-COHEN}

Université Charles-de-Gaulle - Lille 3 